\title{
Applications of New Technologies and New Methods in ZHENG Differentiation
}

\author{
Jianye Dai, ${ }^{1}$ Shujun Sun, ${ }^{1}$ Huijuan Cao, ${ }^{1}$ Ningning Zheng, ${ }^{1}$ Wenyu Wang, ${ }^{1}$ \\ Xiaojun Gou, ${ }^{2}$ Shibing Su, ${ }^{3}$ and Yongyu Zhang ${ }^{1}$ \\ ${ }^{1}$ Research Center for Traditional Chinese Medicine and Systems Biology, Shanghai University of Traditional Chinese Medicine, \\ 1200 Cailun Road, Pudong, Shanghai 201203, China \\ ${ }^{2}$ Key Laboratory of Liver and Kidney Diseases of Ministry of Education, Shuguang Hospital, Institute of Liver Diseases, \\ Shanghai University of Traditional Chinese Medicine, 528 Zhanghen Road, Pudong, Shanghai 201203, China \\ ${ }^{3}$ Research Center for Traditional Chinese Medicine Complexity System, Shanghai University of Traditional Chinese Medicine, \\ 1200 Cailun Road, Pudong, Shanghai 201203, China
}

Correspondence should be addressed to Shibing Su, shibingsu07@163.com and Yongyu Zhang, dryyz@sina.com

Received 12 February 2012; Accepted 21 March 2012

Academic Editor: Aiping Lu

Copyright (c) 2012 Jianye Dai et al. This is an open access article distributed under the Creative Commons Attribution License, which permits unrestricted use, distribution, and reproduction in any medium, provided the original work is properly cited.

\begin{abstract}
With the hope to provide an effective approach for personalized diagnosis and treatment clinically, Traditional Chinese Medicine (TCM) is being paid increasing attention as a complementary and alternative medicine. It performs treatment based on ZHENG (TCM syndrome) differentiation, which could be identified as clinical special phenotypes by symptoms and signs of patients. However, it caused skepticism and criticism because ZHENG classification only depends on observation, knowledge, and clinical experience of TCM practitioners, which is lack of objectivity and repeatability. Scientists have done fruitful researches for its objectivity and standardization. Compared with traditional four diagnostic methods (looking, listening and smelling, asking, and touching), in this paper, the applications of new technologies and new methods on the ZHENG differentiation were systemically reviewed, including acquisition, analysis, and integration of clinical data or information. Furthermore, the characteristics and application range of these technologies and methods were summarized. It will provide reference for further researches.
\end{abstract}

\section{Introduction}

Personalized medicine is looming all over the world, especially following the accomplishment of the Human Genome Project (HGP). Major investments in basic science have created an opportunity for significant progress in clinical medicine. Scientists are developing and using diagnostic tests based on genetics or other molecular mechanisms to better predict patients' responses to targeted therapies [1]. Yet, myriad obstacles must be overcome to achieve these goals.

As a holistic approach attempting to bring the body, mind, and spirit into harmony, TCM may bring personalized medicine to the light in an efficient way. As the essential part of its theory, ZHENG, also called Traditional Chinese Medicine (TCM) syndrome or pattern, is a characteristic profile of all clinical manifestations identified by TCM practitioners and consists of not only the body condition, but also mind and spirit. With the general knowledge of ZHENG and its evolution, TCM emphasizes on early diagnosis and prognosis of diseases, especially preventing its recurrence. In TCM clinical practice, "Treatment based on ZHENG Differentiation" (Bian Zheng Lun Zhi) often gets better effect. For example, He et al. [2] reveal that the effective rate of a combination therapy of two Chinese patent medicines (Glucosidorum Tripterygll Totorum Tablet and Yishenjuanbi Tablet) on rheumatoid arthritis was $53.3 \%$, without ZHENG differentiation. Nevertheless, the effective rate was up to more than $80 \%$, with ZHENG differentiation. Besides, Lu et al. [3] found that the effective rate of biomedical combination therapy (diclofenac, methotrexate, and sulfasalazine) in cold syndrome was much higher than in heat syndrome $(P<0.01)$. After 12-week treatment, the effective rates in patients with cold syndrome and heat syndrome were $51.67 \%$ and $29.09 \%$, respectively, but for 24 -week treatment, it changed to $88.52 \%$ 
and $57.40 \%$. These researches may suggest that treatment based on ZHENG differentiation could improve the specificity and efficiency in both TCM and Western Medicine.

Although TCM has been practiced effectively more than 3000 years, ZHENG differentiation is still argued, because it depended on clinical observation and TCM practitioner's experience, which would be subjective and unrepeatable. Since the success of personalized medicine relies on having accurate diagnostic tests that identify patients who can benefit from targeted therapies [1], a great breakthrough in TCM diagnosis with objectivity and repeatability is needed. For this goal, TCM researchers have currently done fruitful works with beneficial technologies and methods, such as literature mining and system biological analysis. Here, the new technologies and the new methods of applied in ZHENG differentiation were reviewed, at the aspects of acquisition, analysis, and integration of clinical data or information, respectively.

\section{Data Acquisition}

As the saying goes, "one cannot make bricks without straw," a qualitative or a quantitative data is required before ZHENG differentiation. The acquisition of the applicable data using the appropriate technologies or methods is first step.

2.1. Qualitative Data Acquisition. The qualitative data is usually got from literatures, epidemiological questionnaire, and parameters by the traditional four diagnostic methods (looking, listening and smelling, asking, and touching). It could be used to describe the characteristics, distribution, and evolution of ZHENG, further to classification.

2.1.1. Literature Retrieval. Like as that we could have a further view standing on the shoulders of predecessors, literature retrieval is undoubtedly a feasible method for researching ZHENG differentiation. A research [4] was performed to probe into the characteristics of ZHENGs and their elements distributions in polycystic ovary syndrome. Literatures from 1994 to 2009 on ZHENGs were retrieved with keyword search and classified, then especially a database was set up by Excel for further analysis based on the collected data. With the help of those, the frequencies of 36 syndromes and their elements have been analyzed.

It is worthy to say that quality control should be taken seriously in the process of literature collection. And we cannot simply copy the Western standards, for example, Cochrane Statement [5]. An evaluation system for ZHENG differentiation should be established, which is suitable for TCM; otherwise, it will restrict even hold back its development.

2.1.2. Epidemiological Design. Clinical epidemiological study is widely used to acquire data, with the methods of retrospective, cross-sectional, and longitudinal study. With retrospective analysis, diagnostic information of 438 patients with chronic severe hepatitis B (CSHB) was investigated by Peng et al. [6]. The principle signs of TCM syndromes were analyzed by frequency and variable cluster analysis for ZHENG differentiation on three clinical stages. Especially, the research on evolution of "dampness-heat," "spleen deficiency," and "blood stasis" may provide assistance for dynamical ZHENG differentiation.

2.1.3. Improvement of Four Diagnostic Methods. As the most important traditional methods, four diagnostic methods (looking, listening and smelling, asking, and touching) have to be developed. As depended on TCM practitioners' observation and clinical experience, the shortcoming of these methods is absence of objective criteria and repeatability. Yue and Liu [7] and Pang et al. [8] have digitalized tongue images using computer technology, to bring tongue observation to semiquantitative measure. And TCM pulse detector was utilized to improve the accuracy and repeatability of pulse diagnosis and provide the data but just feeling [9].

2.2. Quantitative Data Acquisition. As above data is all acquired by observation and clinical experience, it is not only qualitative and unrepeatable, but also hard to conduct statistical analysis, pattern recognition, and integration with absence of totally digitalization. Therefore, the acquisition of quantitative data is calling for further progress.

2.2.1. "Omics" Technologies. "Omics" consists of genomics, transcriptomics, proteomics, and metabonomics, with the rapid growth of large-scale detection technologies [10]. It directly focuses on biochemistry networks, pathways, metabolites, and molecule targets of whole bodies, at the topto-down views. With the features of nondestructiveness, integrity, multitarget, high-throughput, and digitalization, "Omics" technologies may provide feasibility to investigate ZHENG, which would be characterized by multifactor, multiphenotype, and dynamic state.

Genomics/transcriptomics, also known as global gene expression profiling, is a tool for evaluating gene expression levels of thousands of genes in parallel. Technologies such as gene chip, gene sequencing, and differential display are usually applied. Wu et al. [11] performed genomics to assess the correlation between genetic variations of metabolic genes including PPARD, PPARG, and APM1 and the constitutions. The result suggested that SNP and haplotypes of PPARD, PPARG, and APM1 may underlie the genetic basis of the ZHENG classification. Moreover, gene chip technology was used by $\mathrm{Lu}$ et al. [12] to reveal gene expression profiles in $\mathrm{CD}^{+}{ }^{+} \mathrm{T}$ cells to classify cold and heat syndromes.

Proteomics can be defined as the science and technologies associated with mapping, visualizing, and/or quantitating the expression of all or a majority of the proteins in living systems [13]. Technologies used in proteomics have been around two-dimensional polyacrylamide gels combined with mass spectrometer (MS) or liquid chromatography (LC). With the method of two-dimensional electrophoresis (2DE) combined with matrix-assisted laser desorption/ionization time-of-flight mass spectrometer (MALDI-TOF-MS), Liu et al. [14] evaluated the levels of plasma proteins in health donors and patients with the different ZHENGs of chronic 
hepatitis B. Objective data was provided for ZHENG differentiation and further to suggest the diagnostic standards and guide the clinical treatment. Wu et al. [15] analyzed the plasma from healthy subjects and patients of coronary heart disease. The result found 3 decreased proteins and 6 increased proteins in blood stasis syndrome, compared with normal group. It suggested that fibrinogen and granzyme might be potential diagnostic biomarkers of blood stasis syndrome in coronary heart disease.

Metabonomics is the study of global metabolite profiles in a biological system (isolated cells, tissue, urine, saliva, blood plasma, etc.) under a given set of conditions [16]. Gas chromatography-mass spectrometer (GC-MS), liquid chromatography-mass spectrometer (LC-MS), and nuclear magnetic resonance (NMR) are widely applied in this area. With the technology of GC/MS, Van Wietmarschen et al. [17] have analyzed the plasma metabolism profiles in patients with cold and heat syndromes of rheumatoid arthritis. They classified the two ZHENGs and got seven differential metabolites. Moreover, using UPLC-QTOF-MS, Sun et al. [18] have analyzed urine samples from liverQi invasion patients with premenstrual syndrome. The potential biomarkers and metabolic pathways were found from the metabolic profiles. Furthermore, Liu et al. [19] have detected plasma samples using NMR to explore the dynamic evolution and phase characteristics of phlegm and blood stasis syndromes from the biological features of lipid metabolism.

2.2.2. Physiology and Pathology Detection Technology. Signs, symptoms, and biochemical parameters of patients were collected by Yuan et al. [20] from self-designed questionnaires regarding the four diagnostic methods of TCM. The result suggested that different syndromes have different pathological features. Taking an example, dampness-heat syndrome was characterized by obvious hepatic inflammation, poor synthesis function, and more ascites.

2.2.3. Molecular Biology Detection Technology. The correlation between biochemical indicator and ZHENGs was evaluated by Zhao [21]. Seventy female RA patients with cold or heat syndrome were enrolled in this trial. However, as for the expression of cytokine (TNF- $\alpha$, IL-10, IL-8), clinical inflammatory indexes (ESR), and immune indexes (IgA, IgG, IgM, RF, C3), subjects with heat and cold syndrome showed no significant difference, except CRP.

\section{Data Mining}

For the complexity of biomedicine, it is circumscribed for researches only based on experimental data. Therefore, objective and accurate description of phenomenon and regularity in TCM is getting out from statistical analysis and data mining, drawing assistance from computer technologies. As a multidiscipline fused artificial intelligence, statistics, pattern recognition, and so on, data mining in database is equal to knowledge discovery $[22,23]$, which is initially utilized for genome designator in biomedicine [24].
3.1. Association Rule Mining. Association rule mining is one of the major approaches of data mining and perhaps the most common method of knowledge discovery in unsupervised learning systems [25]. It is used to describe significant associations or correlation relationships among a large set of data items. Especially, Wu et al. [26] associated the gene function from the MEDLNE with TCM literatures. And then they established the relationship between diseases and ZHENGs, combined with validating the relationship between ZHENGs and genes.

3.2. Rough Sets Theory. As a new math tool to deal with ambiguous and uncertain information, rough sets theory introduced by Pawlak [27] is applied to get some decisions and classification. By deleting unrelated or unimportant information, it is able to simplify information on the premise of keeping classification ability unchanged. The information of symptoms and signs from 287 posthepatitic cirrhosis patients were collected by Zhang et al. to explore the application of rough sets theory in TCM ZHENG diagnosis. The result showed that this model was meaningful for the diagnosis, with $83 \%$ coincidence to main six ZHENGs in TCM [28].

3.3. Cluster Analysis. Cluster analysis, an exploring way of classification, could describe a set of multivariate methods and techniques. It is often used to classify data into groups, types, profiles, and so on [29]. With multicenter and largesample survey, two-step cluster analysis was utilized to study the ZHENG distribution rule of essential hypertension by $\mathrm{Gu}$ et al. [30]. Compared with the current ZHENG differentiation criteria, this method could add two more ZHENGs which may be used to reflected etiological factor.

3.4. Bayesian Networks. Bayesian network is a kind of probability network which is based on probabilistic reasoning, with the foundation of Bayes formula. Especially through their ability to coordinate bidirectional probabilistic inferences, Bayesian networks are now considered to be a general representation scheme for uncertain knowledge $[31,32]$. Qu et al. [33] used Bayesian network to classify ZHENGs in 611 depression patients. The ZHENGs of depression were differentiated by various principle or peripheral ZHENGs and their combinations. The ZHENGs described in their study were in line with clinical TCM and might provide a good guidance for treatment.

3.5. Decision Trees. Decision trees are characterized by a logic function which is constant over some box-shaped regions of the $\mathrm{X}$ range. These regions are usually represented by a binary decision tree consisting of nodes and binary splits [34]. It can be applied in the development of ZHENG classification. Zhong et al. [35] developed a method of decision trees combined with association rules to study Qi stagnation syndrome in gastritis, getting satisfactory prediction.

3.6. Artificial Neural Network. With ability to fitting function at any precision, artificial neural network is powerful to use a 
structure similar with cerebrum neural synapse to deal with information. It has been demonstrated successfully in many classification tasks [36]. Neural network model trained by conjugate gradient algorithm was built by Sun et al. [37] to classify ZHENGs of coronary heart disease, with $89.2 \%$ accuracy. The research got satisfactory results and overcame the shortcomings of traditional BP algorithm effectively.

3.7. Principal Component Analysis. Beginning with the interrelation of the variables, principal component analysis based on the dimension reduction is a statistical method that could translate many variables to fewer unrelated integrated variances [38]. Metabonomics based on UPLC/MS had been performed by Lu et al. [39] to study Kidney-Yang deficiency syndrome and therapeutic effect of Rhizoma Drynariae. With PCA, a clear separation of model group and predose group was achieved. The time-dependent regression tendency in Rhizoma Drynariae treatment group from 1 to 15 days was obtained, which provided a visual, overall, and dynamic progress.

3.8. Partial Least Squares Method. Partial least squares (PLSs) method was proposed by Wold, which extracts characteristics based on the principle of maximizing covariance of independent and dependent variable [40]. It makes the characteristics to have much associativity with the dependent variable, improving the precision of the ZHENG classification followed. As clinical samples have more individual variations than animal samples, the supervised methods like PLS are better at concerning the main intergroup difference of clinical samples than unsupervised methods like PCA. Van Wietmarschen et al. [17] used partial least squaresdifferentiation analysis (PLS-DA) to distinguish cold and heat syndromes of RA patients which were not distinguished by PCA, getting satisfactory result of 3-oxo-propionic acid and other differential metabolites.

3.9. Factor Analysis. Factor analysis is used to find the least number of factors to account for the common variance of a large set of statistical expert system variables, excluding variable-specific (unique) variance [41]. It could be applied in analyzing the correlativity of many primitive markers, and then finding out the limited and unobserved potential variance which dominates and explains the correlativity. Multicenter prospective research on TCM ZHENG in 815 cases of unstable angina was conducted by using factors analysis with the nonlinear dimension reduction. Wang et al. [42] suggested that this method could help to classify ZHENG and establish the preliminary diagnostic criteria.

3.10. Structural Equation Modeling. Structural equation modeling is based on statistical methodology to study and deal with complex and multivariable data. This technique allowed for the computation of individual measurement errors associated with the observed variables [43]. What is more, it allows testing of a priori hypotheses about the complex causality between the latent variables of diseases and ZHENGs. Here, the ZHENGs and domain changes of menopause syndrome on samples of 236 women from literature retrieval were identified by exploratory factor analysis. After finding principle ZHENG of Kidney-Yang and KidneyYin deficiency by latent tree, structural equation modeling was applied to confirm the former result [44].

In addition, set pair analysis [45], logistic regression [46], entropy cluster algorithm [47], and support vector machines [48] were applied in ZHENG differentiation with satisfactory results.

\section{Integration of Data or Bioinformation}

An example is shown about how to integrate information. Systems biology approach with the combination of computational analysis and animal experiment was used to investigate this complex issue, ZHENG, in the context of the neuroendocrine immune (NEI) system. By using the methods of literature mining, network analysis, and topological comparison, it was revealed that hormones and immune factors were predominant in the cold and heat syndromes networks, respectively, which were connected by neurotransmitters. In addition, genes related to heat-related diseases are mainly present in the cytokine-cytokine receptor interaction pathway; whereas genes related to cold-related diseases are linked to the neuroactive ligand-receptor interaction pathway. Also, it was in a position to interpret the scientific basis of both ZHENG and associated herbal treatments [49].

The "interaction-network-function" strategy of integration reflecting from "Entity Ontology" to "Relation Ontology" was according to the holism of TCM in methodology.

\section{Summary and Prospect}

With the features of high throughput and multilevel, "Omics," and bioinformatics technologies are appropriate tools to investigate the holistic characteristics of ZHENG differentiation. In order to easily understand technologies and methods, application range, advantages and disadvantages of "Omics," and bioinformatics, it was resumptively summarized in Table 1.

To find the characteristics and pathogenesis of ZHENGs through high throughput and multilevel, qualitative, and qualitative data, the data mining methods were applied. The advantage and the disadvantage of these methods were resumptively summarized in Table 2 .

Given the limitation of single method and single subject, the multidisciplinary such as mathematics, physics, biology, and statistics would be combined underlying the direction of system theory, which may bring ZHENG researches to an objective and quantized way. For example, cold and heat syndrome has been studied with multiple technologies and methods such as "Omics," bioinformatics and laboratory index [50]. And Bayesian network, rough set, and generalize connected coefficient were combined to classify ZHENG in liver cirrhosis [28]. And we advocate that systematically combined the appropriate technologies or methods to establish a characteristic "net-marker" of ZHENG differentiation 
TABLE 1: Brief introduction of "Omics" and bioinformatics.

\begin{tabular}{|c|c|c|c|c|c|}
\hline Omics & Objects & Technologies and methods & Advantages & Disadvantages & Literatures \\
\hline $\begin{array}{l}\text { Genomics } \\
\text { (transcriptomics) }\end{array}$ & $\begin{array}{l}\text { DNA, } \\
\text { mRNA }\end{array}$ & $\begin{array}{l}\text { Gene sequence, } \\
\text { differential display, } \\
\text { subtractive hybridization, } \\
\text { EST, SAGE, chip technology }\end{array}$ & $\begin{array}{l}\text { Gene polymorphism } \\
\text { Susceptibility for } \\
\text { prognosis } \\
\text { and treatment } \\
\text { Completed database } \\
\text { High throughput }\end{array}$ & $\begin{array}{l}\text { Nonassociation to } \\
\text { regulation of life } \\
\text { activities } \\
\text { Nonconsistent } \\
\text { strictly } \\
\text { with mRNA } \\
\text { expression }\end{array}$ & $\begin{array}{l}\text { Wu et al. [11] } \\
\text { Lu et al. [12] }\end{array}$ \\
\hline Proteomics & $\begin{array}{l}\text { Amino acids, } \\
\text { protein }\end{array}$ & $\begin{array}{l}\text { Cleaving isotope-coded affinity tag, } \\
\text { 2D-MS, 2D-HCLP }\end{array}$ & $\begin{array}{l}\text { Performer of life } \\
\text { function }\end{array}$ & $\begin{array}{l}\text { Instability } \\
\text { Variability }\end{array}$ & $\begin{array}{l}\text { Liu et al. [14] } \\
\text { Wu et al. [15] }\end{array}$ \\
\hline Metabonomics & Metabolites & NMR, GC-MS, LC-MS & $\begin{array}{l}\text { Amplified action } \\
\text { Simplicity to detect } \\
\text { Less numbers } \\
\text { Similarities in } \\
\text { different species }\end{array}$ & $\begin{array}{l}\text { Lack of beneficial } \\
\text { supports } \\
\text { Interferences by } \\
\text { physiological } \\
\text { factors }\end{array}$ & $\begin{array}{l}\text { Van Wietmarschen et } \\
\text { al. [17] } \\
\text { Sun et al. [18] } \\
\text { Liu et al. [19] }\end{array}$ \\
\hline Bioinformatics & $\begin{array}{l}\text { Data, } \\
\text { bioinformation }\end{array}$ & $\begin{array}{l}\text { Data mining, network analysis, } \\
\text { topological } \\
\text { comparison, and so on }\end{array}$ & $\begin{array}{l}\text { Totally holism } \\
\text { Exploration of } \\
\text { the potential of } \\
\text { information } \\
\text { Focusing on function } \\
\text { relation }\end{array}$ & $\begin{array}{l}\text { Needing } \\
\text { of } \\
\text { self-development }\end{array}$ & $\operatorname{Li}[49]$ \\
\hline
\end{tabular}

TABLE 2: Brief introduction of data mining methods.

\begin{tabular}{|c|c|c|c|}
\hline Methods & Advantages & Disadvantages & Literatures \\
\hline Logistic regression & Multifunction & Needing of sample size & Luo et al. [46] \\
\hline Bayesian networks & Utilization of incomplete and inaccurate data & $\begin{array}{l}\text { Needing of preceding researches as } \\
\text { guidance }\end{array}$ & Qu et al. [33] \\
\hline Rough sets theory & $\begin{array}{l}\text { Without priori information; simplicity; } \\
\text { handling ambiguous and uncertain } \\
\text { information }\end{array}$ & Needing of self-development & Zhang et al. [28] \\
\hline Association rules mining & Supporting indirect data mining & Nonselectivity; subjectivity & Wu et al. [26] \\
\hline Set pair analysis & Suitability for changing systems & $\begin{array}{l}\text { Handicap in handle relatively precise } \\
\text { problems }\end{array}$ & Li et al. [45] \\
\hline Structural equation modeling & $\begin{array}{l}\text { Analyzing the causality between the latent } \\
\text { variables }\end{array}$ & Needs of 200 samples at least & Chen et al. [44] \\
\hline Cluster analysis & $\begin{array}{l}\text { Minimization errors caused by subjective } \\
\text { judgment }\end{array}$ & $\begin{array}{l}\text { Too much calculation; handicap in } \\
\text { clustering data with multidimensions } \\
\text { and multilevel }\end{array}$ & Gu et al. [30] \\
\hline Decision trees & Handling in nonnumeric data; Simplicity & Maybe misleading & Zhong et al. [35] \\
\hline Principal component analysis & Dimension reduction; holism & Less specificity & Lu et al. [39] \\
\hline Partial least squares method & Specificity & $\begin{array}{l}\text { Handicap in deciding principal } \\
\text { component }\end{array}$ & $\begin{array}{l}\text { Van } \\
\text { Wietmarschen } \\
\text { et al. [17] }\end{array}$ \\
\hline Artificial neural network & Simplicity; nonlinear & $\begin{array}{l}\text { Handicap in obtaining the hidden } \\
\text { information }\end{array}$ & Sun et al. [37] \\
\hline Entropy cluster algorithm & $\begin{array}{l}\text { Little demand on variances' types; analysis on } \\
\text { any statistical dependence of the variances }\end{array}$ & Needing of self-development & Wang et al. [47] \\
\hline Factor analysis & Correction capability; views to latent variables & $\begin{array}{l}\text { Absence of domination and } \\
\text { relationship between primary and } \\
\text { secondary }\end{array}$ & Wang et al. [42] \\
\hline Support vector machine & $\begin{array}{l}\text { Classification without representing the feature } \\
\text { space explicitly }\end{array}$ & $\begin{array}{l}\text { Expressing the more complex prior } \\
\text { information; analyzing limited } \\
\text { samples }\end{array}$ & Yang et al. [48] \\
\hline
\end{tabular}




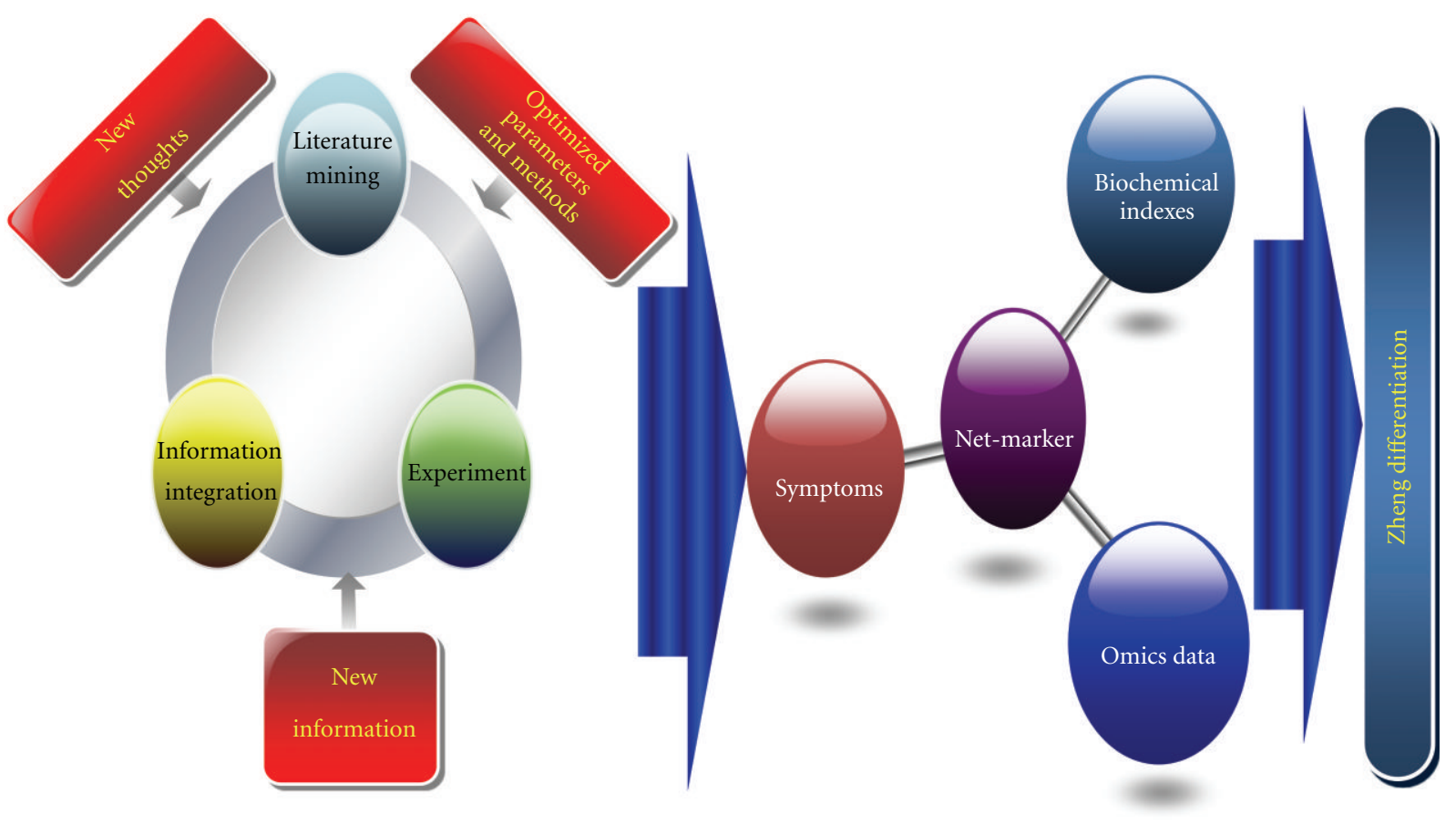

FIGURE 1: Schematic diagram of research approach for ZHENG differentiation.

using clinical signs, syndromes, biochemical indicators, and "Omics" data.

Furthermore, we proposed a ZHENG differentiation research approach bases on a computer-aided "informationexperiment-information” model (Figure 1). By literature mining, researchers firstly could get necessary information to provide ideas, which include clinical syndromes and signs, laboratorial samples using the suitable methods. The ideas could guide new information which comes from experiments and supply validation. Then, analysis and integration of new data will produce further information for ZHENG differentiation.

In this progress, assistances are drawn from computer technologies. Data mining could provide the comprehensive and efficient way to deal with the massive data. Suitable methods with broader vision and optimized parameters could be explored by the objective data, but experience and subjective decision. And then, feedback will be got timely from the experiments by powerful statistical analysis, to guide next ones. Furthermore, the "net-marker" acquired from integration of former results may provide an overall and novel understanding of ZHENG for differentiation. The approach shows many differences to traditional thoughts on feasibility and directivity, reducing blindness and consumption (Figure 1).

In addition, for the clinical transformation in ZHENG differentiation, a further research of dynamic changes of ZHENG is needed. Following the development of highthroughout and noninvasive methods, especially the system biological technologies, may give the support to the dynamically differentiating ZHENG. Furthermore, the TCM information and bioinformation would be combined to make
TCM syndrome network with the dynamic characteristic by bioinformatics and computer technologies.

Following the development of new technologies and new methods, the upgrade of TCM researchers' ability, and the expansion of views on the research of ZHENG differentiation, we all believe that objective and accurate approach would be beneficial to TCM diagnosis and treatment. As a result, TCM may play a more important role in personalized medicine.

\section{Acknowledgments}

This study was supported by National Science and Technology Major Project of China (no. 2012ZX10005001-004) and Leading Academic Discipline Project of Shanghai Municipal Education Commission (no. J50301).

\section{References}

[1] M. A. Hamburg and F. S. Collins, "The path to personalized medicine," The New England Journal of Medicine, vol. 363, no. 4, pp. 301-304, 2010.

[2] Y. He, A. Lu, Y. Zha, and I. Tsang, "Differential effect on symptoms treated with traditional Chinese medicine and western combination therapy in RA patients," Complementary Therapies in Medicine, vol. 16, no. 4, pp. 206-211, 2008.

[3] C. Lu, Q. Zha, A. Chang, Y. He, and A. Lu, "Pattern differentiation in traditional chinese medicine can help define specific indications for biomedical therapy in the treatment of rheumatoid arthritis," Journal of Alternative and Complementary Medicine, vol. 15, no. 9, pp. 1021-1025, 2009.

[4] X. M. Liu and Q. Hua, "Investigation on distributed feature of TCM syndrome and syndrome elements about polycystic 
ovary syndrome from modern literature," Chinese Journal of Information on TCM, vol. 18, no. 2, pp. 34-36, 2011.

[5] J. H. Li, J. L. Wang, and C. Meng, "Present researches and analysis of quality valuation on literatures on clinical traditional Chinese medicine," Chinese Journal of Information on TCM, vol. 15, no. 6, pp. 95-98, 2008 (Chinese).

[6] J. Peng, B. Chen, K. W. Sun et al., "Retrospective analysis of distribution and evolution characteristics of syndrome dampness heat-blood stasis-spleen deficiency of chronic severe hepatitis B," Chinese Journal of Integrated Traditional and Western Medicine on Liver Diseases, vol. 21, no. 3, pp. 135-138, 2011.

[7] X. Q. Yue and Q. Liu, "Analysis of studies on pattern recognition of tongue image in traditional Chinese medicine by computer technology," Journal of Chinese Integrative Medicine, vol. 2, no. 5, pp. 326-329, 2004.

[8] B. Pang, D. Zhang, N. Li, and K. Wang, "Computerized tongue diagnosis based on Bayesian networks," IEEE Transactions on Biomedical Engineering, vol. 51, no. 10, pp. 1803-1810, 2004.

[9] J. Q. Hu, T. Zhao, G. C. Xu et al., "Assessment on the accuracy of four types of pulse by SM-1A TCM pulse detector," World Science and Technology/Modernization of Traditional Chinese Medicine and Materia Medics, vol. 13, no. 1, pp. 74-77, 2011.

[10] J. B. German, D. E. Bauman, D. G. Burrin et al., "Metabolomics in the opening decade of the 21st century: building the roads to individualized health," Journal of Nutrition, vol. 134, no. 10, pp. 2729-2732, 2004.

[11] Y. Wu, Y. Cun, J. Dong et al., "Polymorphisms in PPARD, PPARG and APM1 associated with four types of Traditional Chinese Medicine constitutions," Journal of Genetics and Genomics, vol. 37, no. 6, pp. 371-379, 2010.

[12] C. Lu, C. Xiao, G. Chen et al., "Cold and heat pattern of rheumatoid arthritis in traditional Chinese medicine: distinct molecular signatures indentified by microarray expression profiles in CD4-positive T cell," Rheumatology International, vol. 32, no. 1, pp. 61-68, 2010.

[13] N. G. Anderson and N. L. Anderson, "Twenty years of twodimensional electrophoresis: past, present and future," Electrophoresis, vol. 17, no. 3, pp. 443-453, 1996.

[14] Y. P. Liu, P. Liu, R. Y. Dai et al., "Analysis of plasma proteome from cases of the different traditional Chinese medicine syndromes in patients with chronic hepatitis B," Journal of Pharmaceutical and Biomedical Analysis, vol. 59, pp. 173-178, 2012.

[15] J. H. Wu, Z. C. Ma, Y. Gao, and S. Q. Wang, "Proteomics application in Research related proteins of blood stasis syndrome in coronary heart disease by Proteomics," Chinese Journal of Integrative Medicine on Cardio-/Cerebrovascular Disease, vol. 3, no. 3, pp. 189-191, 2005 (Chinese).

[16] S. Rochfort, "Metabonomics reviewed: a new "omics" platform technology for systems biology and implications for natural products research," Journal of Cerebral Blood Flow \& Metabolism, vol. 68, no. 12, pp. 1813-1820, 2005.

[17] H. Van Wietmarschen, K. Yuan, C. Lu et al., "Systems biology guided by Chinese medicine reveals new markers for sub-typing rheumatoid arthritis patients," Journal of Clinical Rheumatology, vol. 15, no. 7, pp. 330-337, 2009.

[18] P. Sun, G. A. Luo, M. Q. Qiao et al., "Studies on the metabonomics of premenstrual syndrome liver-Qi invasion patient interfered by jingqianping granules," World Science and Technology/Modernization of Traditional Chinese Medicine and Materia Medics, vol. 12, no. 2, pp. 195-201, 2010.

[19] W. H. Liu, X. Z. Yan, L. Zhang, Q. Zhang, L. H. Wang, and F. H. Yu, "Metabonomics study on phlegm and blood stasis evolution of hyperlipidemia and atherosclerosis," Liaoning Journal of Traditional Chinese Medicine, vol. 49, no. 8, pp. 738741, 2008.

[20] J. L. Yuan, H. Zhang, L. Wang et al., "Biochemical characteristics of traditional Chinese medicine syndromes and their elements in patients with hepatitis B cirrhosis," Journal of Chinese Integrative Medicine, vol. 9, no. 4, pp. 374-381, 2011.

[21] L. H. Zhao, C. Xiao, X. P. Yan et al., "Correlation between heat or cold syndrome and cytokine, and laboratory index in women with early rheumatoid arthritis," Acta Universitatis Traditionis Medicalis Sinensis Pharmacologiaeque Shanghai, vol. 20, no. 1, pp. 21-24, 2006.

[22] Y. C. Shen, X. Y. Wang, and Y. M. Cai, "Application and expectation of data mining in traditional Chinese medical research of syndrome and treatment," Chinese journal of Integrated Traditional and Western Medicine, vol. 28, no. 9, pp. 847-850, 2008.

[23] L. Zhu, B. Wu, and C. Cao, "Introduction to medical data mining," Journal of Biomedical Engineering, vol. 20, no. 3, pp. 559-562, 2003.

[24] W. Ding, Y. G. Jiang, Y. P. Song, M. X. Wu, and K. Li, "Data mining application in traditional Chinese medicine," Journal of Mathematical Medicine, vol. 20, no. 3, pp. 403-404, 2007 (Chinese).

[25] M. S. Chen, J. Han, and P. S. Yu, "Data mining: an overview from a database perspective," IEEE Transactions on Knowledge and Data Engineering, vol. 8, no. 6, pp. 866-883, 1996.

[26] Z. H. Wu, X. Z. Zhou, B. Y. Liu, and J. L. Chen, “Text mining for finding functional community of related genes using TCM knowledge," Knowledge Discovery in Database, vol. 3202, pp. 459-470, 2004.

[27] Z. Gong, B. Sun, and D. Chen, "Rough set theory for the interval-valued fuzzy information systems," Information Sciences, vol. 178, no. 8, pp. 1968-1985, 2008.

[28] Y. H. Zhang, P. Liu, Q. Zhang et al., "Bayesian model to traditional Chinese medicine syndrome classification based on the rough set theory and generalized-relating-degree coefficient," Statistics and Decision, vol. 264, pp. 159-161, 2008 (Chinese).

[29] S. T. Leonard and M. Droege, "The uses and benefits of cluster analysis in pharmacy research," Research in Social and Administrative Pharmacy, vol. 4, no. 1, pp. 1-11, 2008.

[30] W. L. Gu, Z. X. Shi, Y. X. Yu, Y. W. Wu, B. W. Lu, and K. K. Hui, "Distribution characteristics of syndrome types in essential hypertension," Journal of Chinese Integrative Medicine, vol. 8, no. 9, pp. 842-847, 2010.

[31] K. B. Korb and A. E. Nicholson, Bayesian Artificial Intelligence, Chapman and Hall/CRC Computer Science and Data Analysis Series, Taylor \& Francis, 2010.

[32] A. Biedermann and F. Taroni, "Bayesian networks for evaluating forensic DNA profiling evidence: a review and guide to literature," Forensic Science International, vol. 6, no. 2, pp. 147157, 2012.

[33] M. Qu, Q. S. Tang, Z. X. Bao, Y. Wang, and L. Ma, "Bayesian network application in traditional Chinese medicine syndrome," Chinese archives of Traditional Chinese Medicine, vol. 26, no. 7, pp. 1497-1498, 2008.

[34] A. Neumann, J. Holstein, J. R. Le Gall, and E. Lepage, "Measuring performance in health care: case-mix adjustment by boosted decision trees," Artificial Intelligence in Medicine, vol. 32, no. 2, pp. 97-113, 2004.

[35] Y. Zhong, X. L. Hu, and J. F. Lu, "Diagnosis in traditional Chinese medicine on gastritis based on relation rules and 
decision trees," Chinese Journal of Information on Traditional Chinese Medicine, vol. 15, no. 8, pp. 97-99, 2008 (Chinese).

[36] P. K. Simpson, Artificial Neural Systems, Pergamon Press, Oxford, UK, 1989.

[37] G. X. Sun, C. Y. Liao, Z. K. Yuan, Z. X. Hu, and T. Ren, "Neural Network Modelsto Traditional Chinese Medicine Syndrome in coronary heart disease based on conjugategradientalgorithm," Chinese Journal of Integrative Medicine on Cardio-/Cerebrovascular Disease, vol. 6, no. 3, pp. 339-340, 2008 (Chinese).

[38] K. J. Friston, C. D. Frith, P. F. Liddle, and R. S. J. Frackowiak, "Functional connectivity: the principal-component analysis of large (PET) data sets," Journal of Cerebral Blood Flow and Metabolism, vol. 13, no. 1, pp. 5-14, 1993.

[39] X. Lu, Z. Xiong, J. Li, S. Zheng, T. Huo, and F. Li, "Metabonomic study on 'Kidney-Yang Deficiency syndrome' and intervention effects of Rhizoma Drynariae extracts in rats using ultra performance liquid chromatography coupled with mass spectrometry," Talanta, vol. 83, no. 3, pp. 700-708, 2011.

[40] K. Tang and T. Li, "Comparison of different partial leastsquares methods in quantitative structure-activity relationships," Analytica Chimica Acta, vol. 476, no. 1, pp. 85-92, 2003.

[41] R. L. Gorsuch, Factor Analysis, Lawrence Erlbaum Associates, Hillsdale,, NJ, USA, 1983.

[42] J. Wang, Q. Y. He, H. X. Li, and Y. T. Fang, "Factor analysis of traditional Chinese medicine syndromes in 815 patients with unstable angina," Journal of Chinese Integrative Medicine, vol. 6, no. 8, pp. 788-792, 2008.

[43] D. Kaplan, Structural Equation Modeling: Foundations and Extensions, Sage, 2006.

[44] R. Q. Chen, C. M. Wong, K. J. Cao, and T. H. Lam, "An evidence-based validation of traditional Chinese medicine syndromes," Complementary Therapies in Medicine, vol. 18, no. 5, pp. 199-205, 2010.

[45] B. Li, R. Xu, F. L. Li, K. Q. Zhao, M. Zhang, and Z. C. Xu, "Study of syndrome differentiation factor of gouty arthritis with blood stasis syndrome based on connection number," Journal of Chinese Integrative Medicine, vol. 7, no. 8, pp. 724$728,2009$.

[46] H. G. Luo, Z. Han, J. X. Li et al., "Study on regularity of Traditional Chinese Medicine Syndrome in precancerous lesion of stomach cancer," China Journal of Traditional Chinese Medicine and Pharmacy, vol. 22, no. 6, pp. 399-401, 2007 (Chinese).

[47] J. Wang, H. X. Li, Z. Q. Sun et al., "Study on syndrome manifestations of Chinese medicine based on complicated algorithm," Journal of Beijing Unveristy of Traditional Chinese Medicine, vol. 29, no. 9, pp. 581-585, 2006 (Chinese).

[48] X. B. Yang, Z. H. Liang, and Y. J. Luo, "P-SVM applications in TCM syndrome classifications," World Science and Technology/Modernization of Traditional Chinese Medicine and Materia Medics, vol. 9, no. 1, pp. 28-31, 2007.

[49] S. Li, "Computational systems biology-based TCM research: a case study of cold/Hot zheng and associated formula," World Science and Technology/Modernization of Traditional Chinese Medicine and Materia Medica, vol. 9, no. 1, pp. 105-111, 2007.

[50] C. Lu, Y. Y. Liu, C. Xiao, M. Jiang, Q. L. Zha, and A. P. $\mathrm{Lu}$, "Biological basis of cold and heat pattern of rheumatoid arthritis in traditional Chinese medicine," World Science and Technology/Modernization of Traditional Chinese Medicine and Materia Medica, vol. 12, no. 5, pp. 814-817, 2010. 


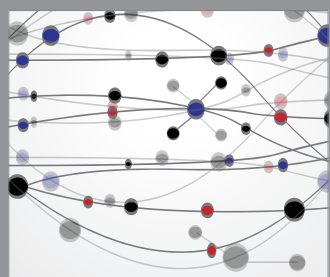

The Scientific World Journal
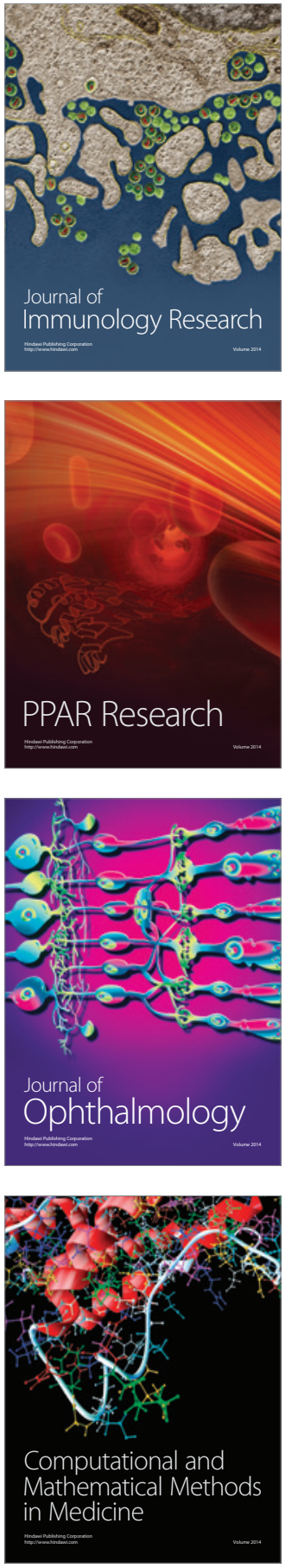

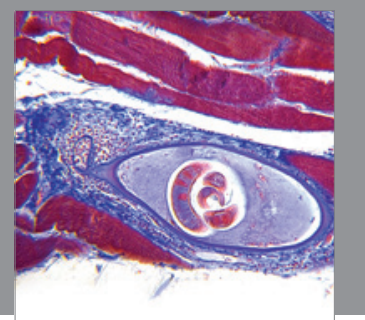

Gastroenterology

Research and Practice
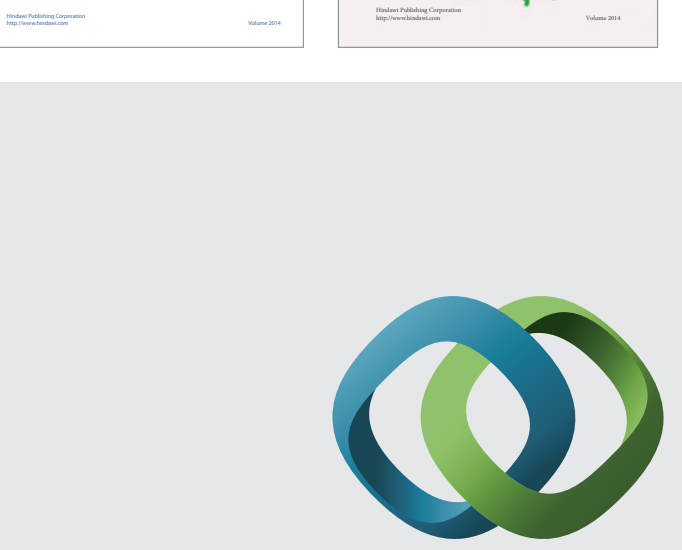

\section{Hindawi}

Submit your manuscripts at

http://www.hindawi.com
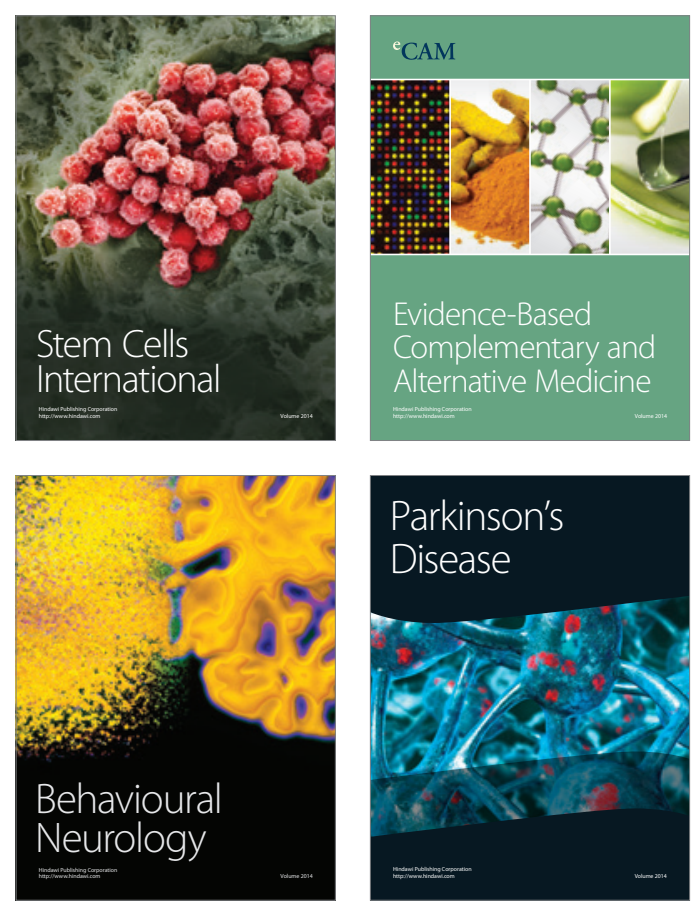

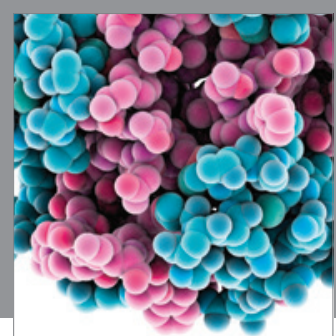

Journal of
Diabetes Research

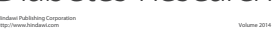

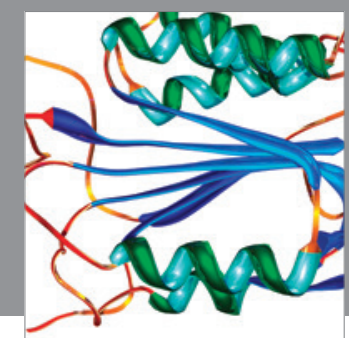

Disease Markers
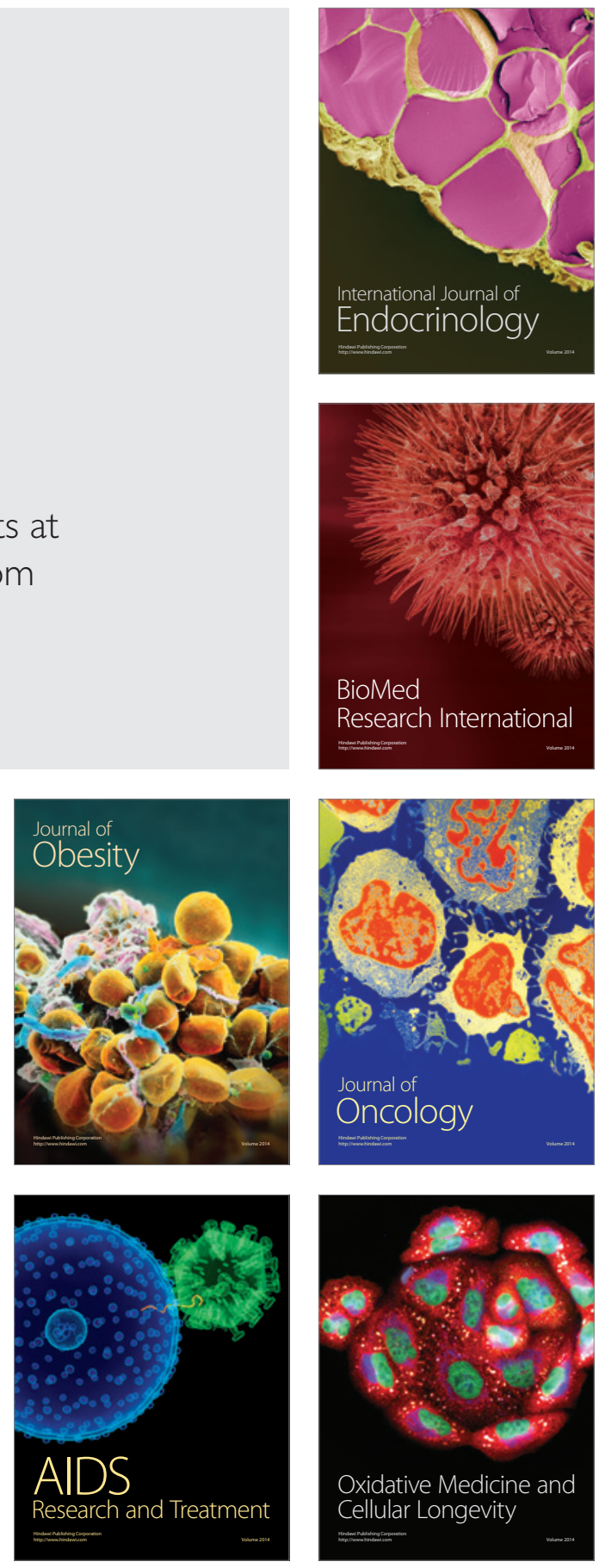